\title{
Global Optimization Method for Robust Pricing of Transportation Networks under Uncertain Demand
}

\author{
Shuaian Wang ${ }^{1}$, Lauren M. Gardner ${ }^{2}$ and S. Travis Waller ${ }^{3}$ \\ ${ }^{1}$ School of Mathematics and Applied Statistics, University of Wollongong, \\ Wollongong, NSW 2522, Australia \\ ${ }^{2}$ School of Civil and Environmental Engineering, The University of New South Wales \\ and NICTA, Sydney, NSW 2052, Australia \\ ${ }^{3}$ School of Civil and Environmental Engineering, The University of New South Wales \\ and NICTA, Sydney, NSW 2052, Australia \\ 1wangshuaian@gmail.com, ${ }^{2}$ gardner@unsw.edu.au, s.waller@unsw.edu.au
}

\begin{abstract}
We extend the existing toll pricing studies with fixed demand to stochastic demand. A new and practical second-best pricing problem with uncertain demand is proposed and formulated as a stochastic mathematical program with equilibrium constraints. In view of the problem structure, we develop a tailored global optimization algorithm. This algorithm incorporates a sample average approximation scheme, a relaxation-strengthening method, and a linearization approach. The proposed global optimization algorithm is applied to three networks: a two-link network, a seven-eleven network and the Sioux-Falls network. The results demonstrate that using a single fixed estimation of future demand may overestimate the future system performance, which is consistent with previous studies. Moreover, the optimal toll obtained by using the mean demand value may not be optimal considering demand uncertainty. The proposed global optimization algorithm explicitly captures demand uncertainty and yields solutions that outperform those without considering demand uncertainty.
\end{abstract}

Keywords: Toll Pricing; Transportation Network Equilibrium; Congestion; Global Optimization

\section{Introduction}

Traffic congestion has become a great concern in heavily populated metropolitan areas [9, 15]. When it is not feasible to increase the capacity of the transportation network, imposing appropriate tolls on roads can reduce traffic congestion because tolls can encourage travelers to seek less direct routes or to travel during a less congested period. Tolls are applied in many cities such as London, Singapore, and Stockholm.

In the literature, the problem of determining tolls to reduce congestion/total travel time is referred to as the toll or congestion pricing problem. The toll pricing problem can be classified as the first and second best. The first-best toll pricing problem assumes that every road or arc in a transportation network can be tolled. The system optimum (SO) can be achieved by setting the toll on a link at the difference between the marginal social cost and the user cost [17]. The second-best toll pricing problem assumes that only a subset of arcs in a transportation network can be tolled because of political reasons and the high cost of setting 
up the toll gantries. Tolls under such a situation generally could not achieve an SO traffic flow and hence are referred to as "second-best".

There are a number of research efforts that are devoted to the toll pricing problem. However, almost all of them calculate the toll based on a single value of travel demand or a deterministic elastic demand relationship [3, 5, 6, 7, 8, 12, 13, 14, 18, 21, 22, 23, 24, 25, 26]. Waller et al., [20] showed that using a single fixed estimation of future demand may overestimate the future system performance. Nagae and Akamatsu [16] and Chen and Subprasom [1] studied the optimal toll on a single private toll road in road franchising considering demand uncertainty. Li et al., [4] examined the toll design for improving the reliability of travel time under uncertain demand. Gardner et al., [2] investigated different techniques for first-best toll pricing with uncertain demand.

We relax the deterministic demand assumption by considering a stochastic demand in the second-best pricing context. The rationale behind using a stochastic demand is threefold. First, the forecasted deterministic demand may not match the real demand. Second, the real demand actually fluctuates day by day, and hence the real demand itself is uncertain. Third, the algorithms developed for models with stochastic demand are also applicable for fixed demand because models with stochastic demand nest the case of fixed demand as a special case. According to the above literature review, maximizing the expected efficiency of a transport network by second-best pricing is a new and practical research topic. The contribution of this paper is as follows. (i) We propose and formulate the second-best pricing problem with uncertain demand. (ii) We design an example to demonstrate that the optimal toll obtained by using the mean demand value may not be optimal considering demand uncertainty. (iii) We develop a tailored global optimization algorithm to address the secondbest pricing problem with uncertain demand. This algorithm incorporates a sample average approximation scheme, a relaxation-strengthening method, and a linearization approach. The proposed global optimization algorithm is applied to three networks.

\section{Notation, Problem Description and Formulation}

The second-best pricing problem with uncertain demand aims to maximize the average network performance in view of the stochastic nature of the demand. It can be formulated as a stochastic mathematical program with equilibrium constraints (MPEC). Before presenting the mathematical model, we list the notation used throughout the paper in Table 1.

In the transportation network $G=(N, A)$ there are a set of toll links represented by $\bar{A} \subseteq A$. The transport authority needs to determine the optimal toll level from a set of given toll levels represented by set $I_{a}, a \in \bar{A}$. The toll at level $i \in I_{a}$ is $\tau_{a}^{i}$. For example $\tau_{a}^{i}$ may be equal to 0.5 USD, 1 USD, 1.5 USD, etc. If no toll is also considered, then we add to set $I_{a}$ a particular level $i$ satisfying $\tau_{a}^{i}=0$. For instance, if $I_{a}=\{0,1,2\}$ and $\tau_{a}^{0}=0, \tau_{a}^{1}=0.5, \tau_{a}^{2}=1$, then the transport authority will choose either toll level 0 (no toll) or level 1 (0.5 USD) or level 2 (1 USD). We define a binary decision variable $z_{a}^{i}$ which equals 1 if and only if toll level $i \in I_{a}$ is levied on link $a \in \bar{A}$, and 0 otherwise. Define toll vector $\mathbf{z}:=\left(z_{a}^{i}, a \in \bar{A}, i \in I_{a}\right)$ that represent the toll setting. Define set $z$ that contains all feasible toll settings:

$$
Z:=\left\{\mathbf{z} \mid z_{a}^{i} \in\{0,1\}, a \in \bar{A}, i \in I_{a} ; \sum_{i \in I_{a}} z_{a}^{i}=1, a \in \bar{A}\right\}
$$




\section{Table 1. Notation}

\begin{tabular}{|c|c|}
\hline Sets & \\
\hline$A$ & Set of all links in the transport network \\
\hline $\bar{A}$ & Set of toll links, $\bar{A} \subseteq A$ \\
\hline$G$ & Set of the transport network, $G=(N, A)$ \\
\hline$I_{a}$ & Set of possible toll levels on link $a \in \bar{A}$. \\
\hline$N$ & Set of nodes in the network \\
\hline$W$ & Set of OD pairs \\
\hline$\hat{\Omega}_{a}$ & Set that contains the slopes and intercepts lines for approximating $v_{a} t_{a}\left(v_{a}\right)$ \\
\hline$\tilde{\Omega}_{a}$ & Set that contains the slopes and intercepts lines for approximating $\int_{0}^{v_{a}} t_{a}(x) d x$ \\
\hline $\bar{\Omega}_{\mathrm{v}}^{s}$ & Set that contains the feasible link flows for scenario $s \in S$ \\
\hline $\bar{\Omega}_{\mathrm{z}}$ & Set that contains all the generated solutions \\
\hline$\Omega$ & Set of demand scenarios. The probability of each scenario is known. \\
\hline$Z^{\bar{N}}$ & Set of $\bar{N}$ candidate solutions \\
\hline Z & Set that contains all feasible toll settings \\
\hline $\mathbf{V}^{\omega}$ & Set of link flows that satisfy the flow conservation equation in scenario $\omega \in \Omega$ \\
\hline$S$ & A sample of the demand with the size $S$ \\
\hline$S^{\prime}$ & A sample of the demand with the size $S^{\prime}$ \\
\hline$W$ & Set of OD pairs \\
\hline \multicolumn{2}{|c|}{ Parameters } \\
\hline$b_{m}^{o \omega}$ & A parameter defined to be $\sum_{(o, d) \in W} q_{o d}^{\omega}$ if $m=o$ and $b_{m}^{o \omega}:=-q_{o m}^{\omega}$ otherwise \\
\hline$q_{o d}^{\omega}$ & Given travel demand for OD pair $(o, d) \in W$ in scenario $\omega \in \Omega$ \\
\hline$M$ & A large number \\
\hline$t_{a}\left(v_{a}\right)$ & Travel time function on link $a \in A$ \\
\hline$\tau_{a}^{i}$ & Toll at level $i \in I_{a}$ \\
\hline$T_{\omega}^{\mathrm{UE}}(\mathbf{0})$ & Total system travel time in demand scenario $\omega \in \Omega$ \\
\hline$T_{\omega}^{\text {so }}$ & Total system optimal travel time in demand scenario $\omega \in \Omega$ \\
\hline \multicolumn{2}{|r|}{ Decision Variables } \\
\hline$z_{a}^{i}$ & $\begin{array}{l}\text { A binary decision variable which equals } 1 \text { if and only if toll level } i \in I_{a} \text { is imposed on } \\
\text { link } a \in \bar{A} \text {, and } 0 \text { otherwise }\end{array}$ \\
\hline $\mathbf{z}$ & Toll vector defined as $\mathbf{z}:=\left(z_{a}^{i}, a \in \bar{A}, i \in I_{a}\right)$ \\
\hline$v_{a}$ & Flow on link $a \in A$ \\
\hline $\mathbf{v}$ & A vector defined as $\mathbf{v}:=\left(v_{a}, a \in A\right)$ \\
\hline$v_{a}^{o \omega}$ & Flow on link $a \in A \quad$ that originate from node $o \in N$ in demand scenario $\omega \in \Omega$ \\
\hline$v_{a}^{\omega}$ & Flow on link $a \in A$ in demand scenario $\omega \in \Omega$ \\
\hline $\mathbf{v}^{\omega}$ & A vector defined as $\mathbf{v}^{\omega}:=\left(v_{a}^{\omega}, a \in A\right)$ \\
\hline $\mathbf{v}^{\omega}(\mathbf{z})$ & UE link flow in demand scenario $\omega \in \Omega$ \\
\hline$v_{a}^{o}$ & Flow on link $a \in A$ that are from node $o \in N$ \\
\hline$\hat{T}_{a}^{s}$ & An auxiliary decision variable, $a \in A, s \in S$ \\
\hline$\tilde{T}_{a}^{s}$ & An auxiliary decision variable, $a \in A, s \in S$ \\
\hline
\end{tabular}




\begin{tabular}{|ll|}
\hline$\hat{T}_{a}^{s}$ & An auxiliary decision variable, $a \in \bar{A}, s \in S$ \\
$\varepsilon$ & A pre-specified tolerance \\
\hline Others & \\
\hline$c^{*}$ & The optimal value of [P] \\
$c_{s}$ & The optimal value of [SAA] \\
$\bar{c}_{s}$ & Mean value of $c_{s}$ \\
$\bar{N}$ & Number of [SAA] models to solve \\
$c^{*}$ & The optimal value of [P] \\
$T_{\omega}^{\text {UE }}(\mathbf{z})$ & Total system travel time in demand scenario $\omega \in \Omega$ when toll vector $\mathbf{z}$ is levied \\
$\Lambda^{\omega}$ & $\begin{array}{l}\text { Savings in total system travel time by applying a toll vector } \mathbf{z} \text { as a ratio of the maximum } \\
\text { possible savings in demand scenario } \omega \in \Omega\end{array}$ \\
\hline
\end{tabular}

We assume that the set of uncertain demand $\Omega$ has a very large number of scenarios and the probability of each scenario is a priori known. In demand scenario $\omega \in \Omega$, the travel demand for OD pair $(o, d) \in W$ is represented by $q_{o d}^{\oplus}$. Let $\mathbf{v}^{\circ}$ represent the vector of link flows in scenario $\omega \in \Omega, \mathbf{v}^{\omega}:=\left(v_{a}^{\omega}, a \in A\right)$. Let $\mathbf{v}^{\oplus}$ represent the set of link flows that satisfy the flow conservation equation. $\mathbf{v}^{\oplus}$ can be formulated as:

$$
\mathbf{V}^{\omega}=\left\{\mathbf{v}^{\omega}\left\{\begin{array}{c}
\sum_{a \in A} v_{a=(m, n)}^{o \omega}-\sum_{a \in A} v_{a=(n, m)}^{o \omega}=b_{m}^{o \omega}, \forall m \in N, \forall o \in N \\
v_{a}^{\omega}=\sum_{o \in N} v_{a}^{o \omega}, \forall a \in A \\
v_{a}^{o \omega} \geq 0, \forall a \in A, \forall o \in N
\end{array}\right\}, \forall \omega \in \Omega\right.
$$

Note that in Eq. (2) we use origin-based link flow formulation rather than the commonly used path flow formulation in most studies on traffic assignment. As will be shown later, this link flow formulation could take advantage of the state-of-the-art mixed-integer linear programming solvers.

Let $T_{\omega}^{\mathrm{UE}}(\mathbf{0})$ be the total system travel time in demand scenario $\omega \in \Omega$ when no toll is levied, $T_{\omega}^{\mathrm{UE}}(\mathbf{z})$ be the total system travel time in demand scenario $\omega \in \Omega$ when toll vector $\mathbf{z}$ is levied, and $T_{\omega}^{\text {so }}$ be the total system optimal travel time in demand scenario $\omega \in \Omega$. Then the relative efficiency for scenario $\omega \in \Omega$ with a toll vector $\mathbf{z}$, represented by $\Lambda^{\omega}(\mathbf{z})$ is defined to be the savings in total system travel time as a ratio of the maximum possible savings (Gardner et al., 2010). Mathematically,

$$
\Lambda^{\omega}(\mathbf{z}):=\frac{T_{\omega}^{\mathrm{UE}}(\mathbf{0})-T_{\omega}^{\mathrm{UE}}(\mathbf{z})}{T_{\omega}^{\mathrm{UE}}(\mathbf{0})-T_{\omega}^{\mathrm{SO}}}
$$

Note that in practice it is rare that $T_{\omega}^{\mathrm{UE}}(\mathbf{0})=T_{\omega}^{\mathrm{sO}}$ and since we are focusing on the secondbest pricing, $\Lambda^{\circ}(\mathbf{z})$ may be strictly less than 1 . Define constant values

$$
\Theta^{\omega}:=\frac{T_{\omega}^{\mathrm{UE}}(\mathbf{0})}{T_{\omega}^{\mathrm{UE}}(\mathbf{0})-T_{\omega}^{\mathrm{SO}}}, \omega \in \Omega
$$

In face of demand uncertainty, the transport authority aims to maximize the expected value of the relative efficiency $\Lambda^{\omega}(\mathbf{z})$ : 


$$
\max _{\mathbf{z} \in \mathbf{Z}} \mathrm{E}_{\omega}\left[\Lambda^{\omega}(\mathbf{z})\right]=\max _{\mathbf{z} \in \mathbf{Z}} \mathrm{E}_{\omega}\left\lceil\frac{T_{\omega}^{\mathrm{UE}}(\mathbf{0})-T_{\omega}^{\mathrm{UE}}(\mathbf{z})}{T_{\omega}^{\mathrm{UE}}(\mathbf{0})-T_{\omega}^{\mathrm{SO}}}\right]=\max _{\mathbf{z} \in \mathbf{Z}} \mathrm{E}_{\omega}\left[\Theta^{\omega}-\frac{T_{\omega}^{\mathrm{UE}}(\mathbf{z})}{T_{\omega}^{\mathrm{UE}}(\mathbf{0})-T_{\omega}^{\mathrm{SO}}}\right]
$$

Therefore we could rewrite the objective as:

$$
c^{*}:=\min _{\mathbf{z} \in Z} \mathrm{E}_{\omega}\left\lceil\frac{T_{\omega}^{\mathrm{UE}}(\mathbf{z})}{T_{\omega}^{\mathrm{UE}}(\mathbf{0})-T_{\omega}^{\mathrm{SO}}}-\Theta^{\omega}\right\rfloor=\min _{\mathbf{z} \in Z} \mathrm{E}_{\omega}\left\lceil\frac{\sum_{a \in A} v_{a}^{\omega}(\mathbf{z}) t_{a}\left(v_{a}^{\omega}(\mathbf{z})\right)}{T_{\omega}^{\mathrm{UE}}(\mathbf{0})-T_{\omega}^{\mathrm{SO}}}-\Theta^{\omega}\right\rfloor
$$

Eq. (6) is the negative of the expected relative efficiency. The link flow vector $\mathbf{v}^{\circ}(\mathbf{z})$ is determined by the lower-level user equilibrium problem:

[UE]

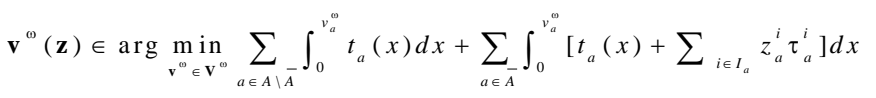

$$
\begin{aligned}
& =\arg \min _{\mathbf{v}^{\circ} \in \mathbf{V}^{\circ}} \sum_{a \in A \backslash \bar{A}} \int_{0}^{v_{a}^{\infty}} t_{a}(x) d x+\sum_{a \in \bar{A}} \sum_{i \in I_{a}} z_{a}^{i} \int_{0}^{v_{a}^{a}}\left[t_{a}(x)+\tau_{a}^{i}\right] d x
\end{aligned}
$$

The "=" in Eq. (7) holds because of the feasible set of toll vectors (1).

Regarding model [P], we have:

Theorem 1: The optimal toll obtained by using the mean demand value may not be optimal considering demand uncertainty.

We will prove this theorem in Section 4.1.

\section{Global Optimization Algorithm}

The model $[\mathrm{P}]$ is a mixed-integer non-convex stochastic optimization problem. There are three difficulties for addressing [P]. First, the cardinality of the uncertain demand scenarios $\Omega$ may be very large. For example, if there are 5 scenarios for each origin-destination (OD) pair, and there are 100 OD pairs, then the cardinality of $\Omega$ is $5100 \approx 8 \times 1069$ and it would be impossible to evaluate or optimize the weighted sum of so many scenarios; if the demand distribution is continuous, then there are an infinite number of scenarios, and the expectation involves multi-dimensional integration, which is intractable. Second, even if there is only one demand scenario, $[\mathrm{P}]$ is still a non-convex optimization problem where the relationship between the toll vector and link flow is implicitly defined by the UE problem. Third, the problem has both discrete decision variables and continuous decision variable, complicating gradient-descent based algorithms.

To overcome the first difficulty, we apply the sample average approximation approach that obtains a good candidate solution along with the statistical estimate of its optimality gap. The second difficulty is addressed by proposing a tailored relaxation-strengthening global optimization algorithm. The third difficulty is overcome by designing a linear approximation scheme that takes advantage of the convexity of the formulation and state-of-the-art mixedinteger linear programming solvers.

\subsection{Sample Average Approximation}

The sample average approximation (SAA) method is an approach for solving stochastic optimization problems by using Monte Carlo simulation. In this technique the objective function of the stochastic program is approximated by a sample average estimate derived from a random sample. The resulting sample average approximating problem is then solved 
by deterministic optimization approaches. This process is repeated with different samples to obtain a good candidate solution along with the statistical estimate of its optimality gap [11, 19].

To apply the SAA method, we first generate $s$ independent and identically distributed observations of the uncertain demand scenario from the support $\Omega$ according to the joint probability mass function or probability density function. These $s$ scenarios are denoted by $1,2 \ldots S$. Let $S:=\{1,2 \cdots S\}$ and the probability of each scenario $s \in S$ is $1 / S$. Therefore, we use a new distribution function with $s$ scenarios of equal occurrence probability to approximate the original uncertain demand whose support has an exponential or an infinite cardinality. The SAA model could be formulated as:

$$
\left.c_{s}:=\min _{\mathbf{z} \in Z} \sum_{s \in S} \frac{1}{S} \mid \frac{\sum_{a \in A} v_{a}^{s}(\mathbf{z}) t_{a}\left(v_{a}^{s}(\mathbf{z})\right)}{T_{s}^{\mathrm{UE}}(\mathbf{0})-T_{s}^{\mathrm{SO}}}-\Theta^{s}\right\rfloor
$$

where

$$
\mathbf{v}^{s}(\mathbf{z}) \in \arg \min _{\mathbf{v}^{s} \in \mathbf{V}^{s}} \sum_{a \in A \backslash \bar{A}} \int_{0}^{v_{a}^{s}} t_{a}(x) d x+\sum_{a \in \bar{A}} \sum_{i \in I_{a}} z_{a}^{i} \int_{0}^{v_{a}^{s}}\left[t_{a}(x)+\tau_{a}^{i}\right] d x
$$

The optimal value $c_{s}$ to model [SAA] is actually a random variable depending on the set $S$. The expected value of $c_{s}$ is no greater than $c^{*}$, namely, $\left.\mathrm{EW}_{s}\right] \leq c^{*}$ (Mak et al., 1999). Consequently, we can generate ${ }^{\bar{N}}$ independent samples of the uncertain demand, each of size $S$, and obtain ${ }^{\bar{N}}$ optimal objective values of model [SAA], denoted by $c_{s}^{n}, n=1,2, \cdots, \bar{N}$. A statistical lower bound for $c^{*}$ can be estimated by $\bar{c}_{s}:=\sum_{n=1}^{\bar{N}} c_{s}^{n} / \bar{N}$. Note that ${ }^{c_{s}}$ is also a random variable. Let $\operatorname{Var}\left(c_{s}^{n}\right)$ be the sample variance of $c_{s}^{n}, n=1,2, \cdots, \bar{N}$. When $\bar{N}$ is large (e.g., 20), $\bar{c}_{s}$ can be considered as normally distributed $\left.\operatorname{Normal}\left(\mathrm{E}_{s}\right], \operatorname{Var}\left(c_{s}^{n}\right) / \bar{N}\right)$ (Strictly speaking, $\left.\left(\bar{c}_{s}-\mathrm{E} w_{s}\right]\right) / \sqrt{\operatorname{Var}\left(c_{s}^{n}\right) / \bar{N}}$ has a t-distribution with $\bar{N}-1$ degrees of freedom). Therefore, in practice we can consider $L B:=\bar{c}_{s}-3 \times \sqrt{\operatorname{Var}\left(c_{s}^{n}\right) / \bar{N}}$ as a stochastic lower bound for the optimal value $c^{*}$ of model $[\mathrm{P}]$ according to the $3 \sigma$ rule (the probability that $L B$ is a lower bound is $99.86 \%$ ).

A total of $\bar{N}$ toll vectors $\mathrm{z}$ are obtained after solving the $\bar{N}$ [SAA] models with different samples. It is possible that some of the toll vectors are identical. We can choose the one with the lowest objective value $c_{s}^{n}$, denoted by $\mathbf{z}^{*}$, for deriving an upper bound as follows. First, a new sample $S^{\prime}$, whose size denoted by $S^{\prime}$ is much larger than $S$, is generated. Then we compute the cost, denoted by $c_{s^{\prime}}$, with fixed toll vector $\mathbf{z}^{*}$ for each scenario $s^{\prime} \in S^{\prime}$. Since $S^{\prime}$ is very large, $U B:=\bar{c}_{s^{\prime}}:=\sum_{s^{\prime} \in S^{\prime}} c_{s^{\prime}} / S^{\prime}$ can be considered as an upper bound for $c^{\prime \prime}$.

The aforementioned approach is the standard SAA procedure in the literature. When applying the standard SAA procedure for continuous optimization problems, the obtained solution is likely to be a good one, however, the possibility that it is optimal is generally 0 . However, in our problem the toll vector is discrete in that it must be chosen from a finite set of candidate toll vectors (although the cardinality of the set is exponential). Therefore, we tailor the SAA approach to our problem setting, and design an improved approach. First, to obtain the upper bound, we evaluate all the total of $\bar{N}$ toll vectors $\mathbf{z}$, denoted by the set $Z^{\bar{N}}$, 
that are obtained after solving the $\bar{N}$ [SAA] models with different samples. For each $\mathbf{z} \in Z^{\bar{N}}$, we generate a new large sample $S^{\prime}$ and compute the resulting expected cost. The toll vector with the lowest cost is implemented and its expected cost is the best upper bound $U B$. Although the computational efforts will be larger, it is worthwhile considering that (i) setting the toll is a long-term decision; (ii) the computational efforts increase at most linearly with $\bar{N}$; and (iii) the most computational efforts lies in solving the $\bar{N}$ [SAA] models.

The lower bound can also be strengthened. In fact, we only need a lower bound for the candidate solutions in set $Z \backslash Z^{\bar{N}}$ as we have already evaluated all the solutions in set $z^{\bar{N}}$. Therefore, we again solve the model [SAA] with another ${ }^{\bar{N}}$ independent samples where $\mathbf{z} \in Z \backslash Z^{\bar{N}}$, and derive a statistical lower bound $L B$. To exclude $Z^{\bar{N}}$ from $Z$, the following constrains are added to [SAA]:

$$
\sum_{a \in \bar{A}_{A} \in I_{a}, \bar{z}_{a}^{i}=0} z_{a}^{i}+\sum_{a \in \bar{A}} \sum_{A \in I_{a}, \bar{z}_{a}^{i}=1}\left(1-z_{a}^{i}\right) \geq 1, \forall \tilde{z}_{a}^{i} \in Z^{\bar{N}}
$$

If $L B \geq U B$, then we are sure that the obtained solution is optimal with a probability of at least $99.86 \%$ (single-sided $3 \sigma$ rule). Otherwise, the optimality gap does not exceed $U B-L B$ with a probability of at least $99.86 \%$.

\subsection{A Relaxation-Strengthening Global Optimization Method}

[SAA] is a mixed-integer programming (MIP) model that is non-convex. To attack the non-convexity of the UE constraints, we first relax [SAA] as

[MIP-relaxed]

$$
c_{S}^{\mathrm{Relax}}:=\min _{\boldsymbol{z} \in Z, \mathbf{v}^{s} \in \mathbf{V}^{v}} \sum_{s \in S} \frac{1}{S}\left\lfloor\frac{\sum_{a \in A} v_{a}^{s} t_{a}\left(v_{a}^{s}\right)}{T_{s}^{\mathrm{UE}}(\mathbf{0})-T_{s}^{\mathrm{SO}}}-\Theta^{s}\right\rceil
$$

Note that in Eq. (11) we use $v_{a}^{s}$ rather than $v_{a}^{s}(\mathbf{z})$ because the constraint (9) is removed. We would impose the constraint (9) dynamically. The algorithm is:

\section{Algorithm 1:}

Step 0: Define a set $\bar{\Omega}_{\mathrm{z}}:=\varnothing$ that will contain all the generated toll vector solutions. Define sets $\bar{\Omega}_{v}^{s}:=\varnothing, s \in S$, that will contain the feasible link flows for scenario $s \in S$.

Step 1: Solve [MIP-relaxed] with the constraints:

$$
\begin{aligned}
& \sum_{a \in A \backslash \bar{A}} \int_{0}^{v_{a}^{s}} t_{a}(x) d x+\sum_{a \in \bar{A}} \sum_{i \in I_{a}} z_{a}^{i} \int_{0}^{v_{a}^{s}}\left[t_{a}(x)+\tau_{a}^{i}\right] d x \leq \sum_{a \in A \backslash \bar{A}} \int_{0}^{\tilde{v}_{a}^{s}} t_{a}(x) d x+\sum_{a \in \bar{A}} \sum_{i \in I_{a}} z_{a}^{i} \int_{0}^{\tilde{v}_{a}^{s}}\left[t_{a}(x)+\tau_{a}^{i}\right] d x \\
& \forall s \in S, \forall \tilde{\mathbf{v}}^{s} \in \bar{\Omega}_{v}^{s}
\end{aligned}
$$

Note that the constraint (12) is valid due to the definition of $v_{a}^{s}(\mathbf{z})$ in Eq. (9). If the optimal solution $z^{\text {opt }}$ coincides with one of the solutions in $\bar{\Omega}_{z}$, output $z^{\text {opt }}$ and stop. Otherwise set $\bar{\Omega}_{\mathbf{z}}:=\bar{\Omega}_{\mathbf{z}} \cup \mathbf{z}^{\text {opt }}$. Solve [UE] by setting $\mathbf{z}$ at $\mathbf{z}^{\text {opt }}$, and obtain the link flow $\mathbf{v}^{s}\left(\mathbf{z}^{\text {opt }}\right)$ for each scenario $s \in S$. Set $\bar{\Omega}_{v}^{s}:=\bar{\Omega}_{v}^{s} \cup \mathbf{v}^{s}\left(\mathbf{z}^{\mathrm{opt}}\right)$. Repeat Step 1 .

Theorem 2: When Algorithm 1 stops, $\mathbf{z}^{\text {opt }}$ is the optimal solution. 
Proof: When $\mathbf{z}^{\text {opt }}$ is generated a second time, its UE flow already exists in $\bar{\Omega}_{v}^{s}$. The corresponding constraints (12) would ensure that the link flow for [MIP-relaxed] is the UE link flow. That is, [MIP-relaxed] with constraints (12) is as tight as [SAA] for solution $\mathbf{z}^{\text {opt }}$. For another solution $\mathbf{z} \in Z$ and $\mathbf{z} \neq \mathbf{z}^{\text {opt }}$, [MIP-relaxed] with constraints (12) is a relaxation of [SAA] if the UE link flow of $\mathbf{z} \in Z$ does not exist in $\bar{\Omega}_{v}^{s}$, and is as tight as [SAA] if the UE link flow of $\mathbf{z} \in Z$ exists in $\bar{\Omega}_{v}^{s}$. Therefore, the objective function value of (11) for $\mathbf{z} \in Z$ and $\mathbf{z} \neq \mathbf{z}^{\text {opt }}$ is not greater than the corresponding value at UE. As the objective value of $\mathbf{z}^{\text {opt }}$ whose link flow is at UE is not greater than the objective value of other solutions whose link flow is relaxed or at $\mathrm{UE}, \mathbf{z}^{\text {opt }}$ is optimal.

Theorem 3: Algorithm 1 terminates in a finite number of iterations.

Proof: This theorem holds trivially because the cardinality of $z$ is finite.

Note that the advantage of dynamically imposing constraint (12) rather than using the variational inequality (VI) formulation [10] is that constraint (12) is convex.

\subsection{A Linearization Approach}

[MIP-relaxed] is a mixed-integer nonlinear programming model. However, its linear programming relaxation is convex. To take advantage of state-of-the-art mixed-integer linear programming solvers, we linearize the nonlinear terms and use linear constraints to approximate them.

The nonlinear term $v_{a} t_{a}\left(v_{a}\right)$ in the objective function (11) can be linearized as shown in Figure 1. The first approximation line is generated from $v_{a}=0$, and the slope is determined such that the maximum gap between the approximation line and $v_{a} t_{a}\left(v_{a}\right)$ when ${ }^{v_{a}}$ varies from the first intersection point $(0,0)$ to the second intersection point equals a pre-specified tolerance level $\varepsilon$. Note that $\varepsilon$ is not a decision variable and the value of the nonnegative $\varepsilon$ does not affect the precision of the final solution. However, one should set an appropriate value of $\varepsilon$ : a too small $\varepsilon$ leads to too many approximation lines, whereas a too large $\varepsilon$ results in low approximation quality. The second approximation line is generated from the second intersection point. This process is repeated until the end point $\left(v_{a}^{\max }, v_{a}^{\max } t_{a}\left(v_{a}^{\max }\right)\right)$ is reached. It should be mentioned that $v_{a}^{\max }$ can be set as the total maximum demand (upper bound of the support) of all OD pairs. Define set $\hat{\Omega}_{a}, a \in A$ that contain the slopes and intercepts lines for approximating $v_{a} t_{a}\left(v_{a}\right)$. The objective function (11) can be formulated as a mixed-integer linear programming (MILP) model by introducing auxiliary decision variables $\hat{T}_{a}^{s}$ :

[MILP-relaxed]

$$
c_{s}^{\text {LP-Relax }}:=\min _{z \in Z, v^{s} \in \mathbf{V}^{s}, \hat{T}_{a}^{s}} \sum_{s \in S} \frac{1}{S}\left\lfloor\frac{\sum_{a \in A} \hat{T}_{a}^{s}}{T_{s}^{\mathrm{UE}}(\mathbf{0})-T_{s}^{\mathrm{SO}}}-\Theta^{s}\right\rceil
$$

subject to

$$
\hat{T}_{a}^{s} \geq k \times v_{a}^{s}+b, \forall a \in A, \forall s \in S, \forall(k, b) \in \hat{\Omega}_{a}
$$

Constraints (12) can be linearized similarly. Note that the right-hand side of constraints (12) is already a linear function of $\mathbf{z}$. To linearize the left-hand side, define set $\tilde{\Omega}_{a}, a \in A$ 
that contain the slopes and intercepts of lines for approximating $\int_{0}^{v_{a}} t_{a}(x) d x$. After introducing auxiliary decision variables $\tilde{T}_{a}^{s}$ and $\hat{T_{a}^{s}}$, constraints (12) are linearized as:

$$
\begin{gathered}
\tilde{T}_{a}^{s} \geq \text { slope } \times v_{a}^{s}+\text { intercept }, \forall a \in A, \forall s \in S, \forall(\text { slope, intercept }) \in \tilde{\Omega}_{a} \\
\hat{T}_{a}^{s} \geq v_{a}^{s} \tau_{a}^{i}-M\left(1-z_{a}^{i}\right), \forall a \in \bar{A}, \forall s \in S, \forall i \in I_{a} \\
\sum_{a \in A} \tilde{T}_{a}^{s}+\sum_{a \in \bar{A}} \hat{T}_{a}^{s} \leq \sum_{a \in A \backslash \bar{A}} \int_{0}^{v_{a}^{s}} t_{a}(x) d x+\sum_{a \in \bar{A}} \sum_{i \in I_{a}} z_{a}^{i} \int_{0}^{\tilde{v}_{a}^{s}}\left[t_{a}(x)+\tau_{a}^{i}\right] d x, \forall s \in S, \forall \tilde{\mathbf{v}}^{s} \in \bar{\Omega}_{v}^{s}
\end{gathered}
$$

As a result, [MILP-relaxed] with constraints (14)-(17) is a MILP model and can be solved by state-of-the-art solvers such as CPLEX.

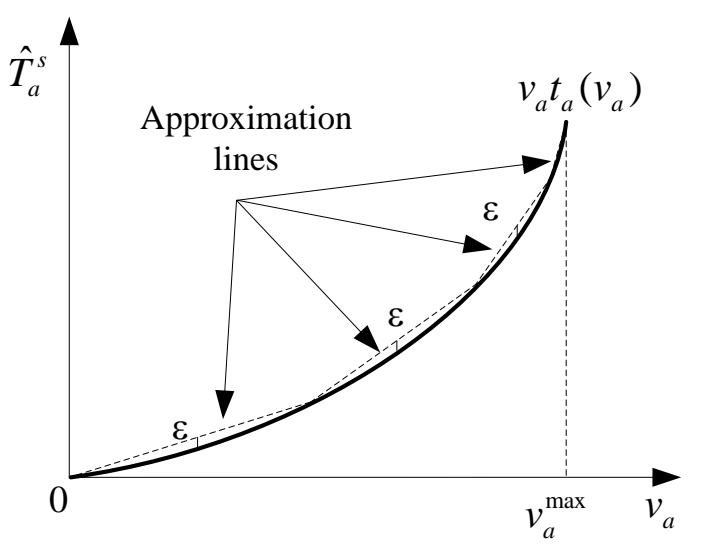

Figure 1. Linearization

\section{Computational Experiments}

The proposed model and algorithm are applied to three networks: one is a simple two-link network; the second network has seven nodes and 11 links and is hence named seven-eleven network; the third network is the Sioux-Falls network. The purpose of the first simple twolink network is to show how the algorithm works and the aim of the seven-eleven network and the Sioux-Falls network is to demonstrate the applicability of the proposed models to more complex cases.

A personal computer with Intel Core (TM) Duo 2.7 GHz CPU, 4 GB RAM, and Windows 7 Professional operating system is used for all tests. The algorithms are coded with $\mathrm{C}++$, calling CPLEX 12.1 to solve the MILP problems.

\subsection{A Two-Link Example}

We first consider a simple two-link example shown in Figure 2 to exemplify the importance of incorporating demand uncertainty in modeling and the process of the relaxation-strengthening global optimization algorithm. The uncertain demand set has two scenarios: $\Omega=\left\{\omega_{1}, \omega_{2}\right\}, q_{1,2}^{\omega_{1}}=15600$ with a probability of $2 / 3$, and $q_{1,2}^{\omega_{2}}=7800$ with a probability of $1 / 3$. The link travel time functions are defined as follows:

$$
\begin{aligned}
& t_{1}\left(v_{1}\right)=6 \times\left[1+0.15\left(v_{1} / 2000\right)^{4}\right] \\
& t_{2}\left(v_{2}\right)=4 \times\left[1+0.15\left(v_{2} / 8000\right)^{4}\right]
\end{aligned}
$$


The transport authority considers imposing toll on link 2 at a value from $\{0,0.25,0.5,0.75,1,1.25,1.5,1.75\}$.

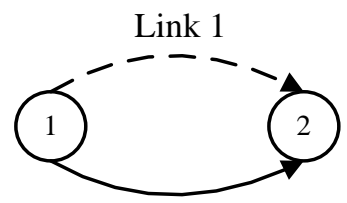

Toll Link 2

\section{Figure 2. A Two-Link Example}

We first address a deterministic model where the uncertain demand is replaced with its average value. In other words, we assume that the demand from node 1 to node 2 is $q_{1,2}^{\omega_{1}} \times(2 / 3)+q_{1,2}^{\omega_{2}} \times(1 / 3)=13000$. The results show that the optimal toll is 1.5 and the relative efficiency $\Lambda=99.5 \%$.

We then consider the stochastic model. Since there are only two demand scenarios, we could enumerate them in the model. That is, the set $S=\Omega=\left\{\omega_{1}, \omega_{2}\right\}$ and the objective function is:

$$
\max _{\mathbf{z} \in Z} \mathrm{E}_{\omega}\left[\Lambda^{\omega}(\mathbf{z})\right]=\frac{2}{3} \times \frac{T_{\omega_{1}}^{\mathrm{UE}}(\mathbf{0})-T_{\omega_{1}}^{\mathrm{UE}}(\mathbf{z})}{T_{\omega_{1}}^{\mathrm{UE}}(\mathbf{0})-T_{\omega_{1}}^{\mathrm{SO}}}+\frac{1}{3} \times \frac{T_{\omega_{2}}^{\mathrm{UE}}(\mathbf{0})-T_{\omega_{2}}^{\mathrm{UE}}(\mathbf{z})}{T_{\omega_{2}}^{\mathrm{UE}}(\mathbf{0})-T_{\omega_{2}}^{\mathrm{SO}}}
$$

In the relaxation-strengthening global optimization algorithm, the first iteration yields solution $\mathbf{z}^{\text {opt }}=\{(1,0,0,0,0,0,0,0)\}$, that is, no toll is imposed. Note that in the first iteration since no constraints (12) exists, the model actually aims to find the SO flows. Therefore any $\mathbf{z} \in Z$ is optimal in this iteration. We then compute the corresponding link flows at UE when the toll is fixed at $\mathbf{z}^{\text {opt }}$ for each of the two demand scenarios, and update the set $\bar{\Omega}_{v}^{s}$. In the second iteration, solution $(0,0,0,0,0,0,1,0)$ is obtained; the third iteration yields solution $(0,0,0,0,0,1,0,0)$; the fourth iteration yields solution $(0,0,0,0,0,1,0,0)$ which is identical to the one obtained in the third iteration. Therefore this solution is optimal.

The optimal toll considering demand uncertainty is hence 1.25 , and the resultant relative efficiency is $85.1 \%$. However, if we set the toll at 1.5 , which is the one obtained by only considering the mean demand, the resultant relative efficiency is $78.0 \%$, which is smaller than $85.1 \%$. This example clearly demonstrates the importance of incorporation of demand uncertainty in congestion pricing. Moreover, this example has actually proved Theorem 1.

We also find that using the mean demand value, we set the toll at 1.5 and the relative efficiency $\Lambda=99.5 \%$. However, if demand uncertainty is considered, the average relative efficiency with the toll of 1.5 is only $78.0 \%$. Hence, this example supports the observation in Waller et al. (2001), that is, using a single fixed estimation of future demand may overestimate the future system performance.

Table 2. Importance of Considering Demand Uncertainty in the Two-Link Example

\begin{tabular}{|c|c|c|}
\hline Strategy & Use the mean demand & Consider demand uncertainty \\
\hline Optimal toll & 1.5 & 1.25 \\
\hline Relative efficiency & $78.0 \%$ & $85.1 \%$ \\
\hline
\end{tabular}




\subsection{The Seven-Eleven Network}

The seven-eleven network, as shown in Figure 3, has 7 nodes and 11 links. There are 4 OD pairs tabulated in Table 3 with their respective average demand. The demand of different OD pairs are independent and the demand of each OD pair has three realizations of equal probability: average value as shown in Table 3, average value multiplied by $110 \%$, and average value multiplied by $90 \%$. The travel time function on each link follows the Bureau of Public Roads (BRP) type function:

$$
t_{a}\left(v_{a}\right)=t_{a}^{0}\left(1.0+0.15\left(\frac{v_{a}}{C_{a}}\right)^{4}\right), a \in A
$$

where $t_{a}^{0}$ denote the free flow travel time and $C_{a}$ is the capacity of each link. The specific value of $t_{a}^{0}$ and $C_{a}$ on each link are provided in Table 4 . The set of toll links $\bar{A}=\{1,2,3,4,11\}$, and possible toll levels $I_{a}=\{0,1,2\}$, and $\tau_{a}^{0}=0, \tau_{a}^{1}=10, \tau_{a}^{2}=20$ for all $a \in \bar{A}$.

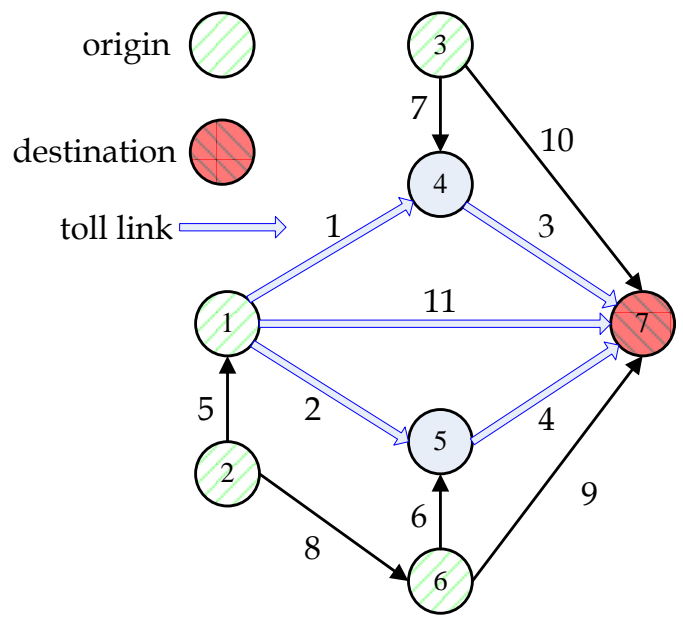

Figure 3. Seven-eleven Network

Table 3. OD Demand

\begin{tabular}{|c|c|}
\hline OD Pair & $\begin{array}{c}\text { Average travel Demand } \\
\text { (vehicle/hour) }\end{array}$ \\
\hline $1 \rightarrow 7$ & 6000 \\
\hline $2 \rightarrow 7$ & 5000 \\
\hline $3 \rightarrow 7$ & 5000 \\
\hline $6 \rightarrow 7$ & 4000 \\
\hline
\end{tabular}

We generate $\bar{N}=10$ independent samples of the uncertain demand, each of size $S=10$. Therefore $\bar{N}$ [SAA] models are solved, and we obtain $\bar{N}$ toll design solutions. For each toll design solution, we generate another sample of size $S^{\prime}=1000$ and calculate the average saving. The best solution $\left(z_{1}^{*}, z_{2}^{*}, z_{3}^{*}, z_{4}^{*}, z_{11}^{*}\right)=(2,0,0,2,0)$ is chosen (that is, a toll of 20 is imposed on links 1 and 4, and no toll is imposed on links 2, 3, and 11) whose average relative efficiency $94.5 \%$ is a lower bound for the problem. 
We then generate another $\bar{N}=10$ independent samples of the uncertain demand, each of size $s=10$. Therefore another $\bar{N}$ [SAA] models where the 10 evaluated toll design solutions are excluded, are solved. The mean of the relative efficiency derived from the [SAA] models is $84.8 \%$, the standard deviation is $1.6 \%$, and therefore an upper bound is $89.8 \%$. Therefore, if we exclude the solution $\left(z_{1}^{*}, z_{2}^{*}, z_{3}^{*}, z_{4}^{*}, z_{11}^{*}\right)$, then with a probability of at least $99.86 \%$ the average relative efficiency cannot exceed $89.8 \%$. However, the average relative efficiency of the solution $\left(z_{1}^{*}, z_{2}^{*}, z_{3}^{*}, z_{4}^{*}, z_{11}^{*}\right)$ is $94.5 \%$. Therefore, $\left(z_{1}^{*}, z_{2}^{*}, z_{3}^{*}, z_{4}^{*}, z_{11}^{*}\right)$ is the optimal solution with a probability of at least $99.86 \%$.

Table 4. Parameters in Link Travel Time Functions

\begin{tabular}{|c|c|c|}
\hline $\begin{array}{c}\text { Link No. } \\
a\end{array}$ & $\begin{array}{c}\text { Free-flow travel time } \\
\text { (seconds) } \\
t_{a}^{0}\end{array}$ & $\begin{array}{c}\text { Capacity (Vehicles/hour) } \\
C_{a}\end{array}$ \\
\hline 1 & 60 & 4000 \\
\hline 2 & 50 & 4000 \\
\hline 3 & 60 & 4000 \\
\hline 4 & 70 & 4000 \\
\hline 5 & 60 & 2000 \\
\hline 6 & 10 & 2000 \\
\hline 7 & 50 & 3000 \\
\hline 8 & 100 & 3000 \\
\hline 9 & 110 & 4000 \\
\hline 10 & 110 & 4000 \\
\hline 11 & 150 & 4000 \\
\hline
\end{tabular}




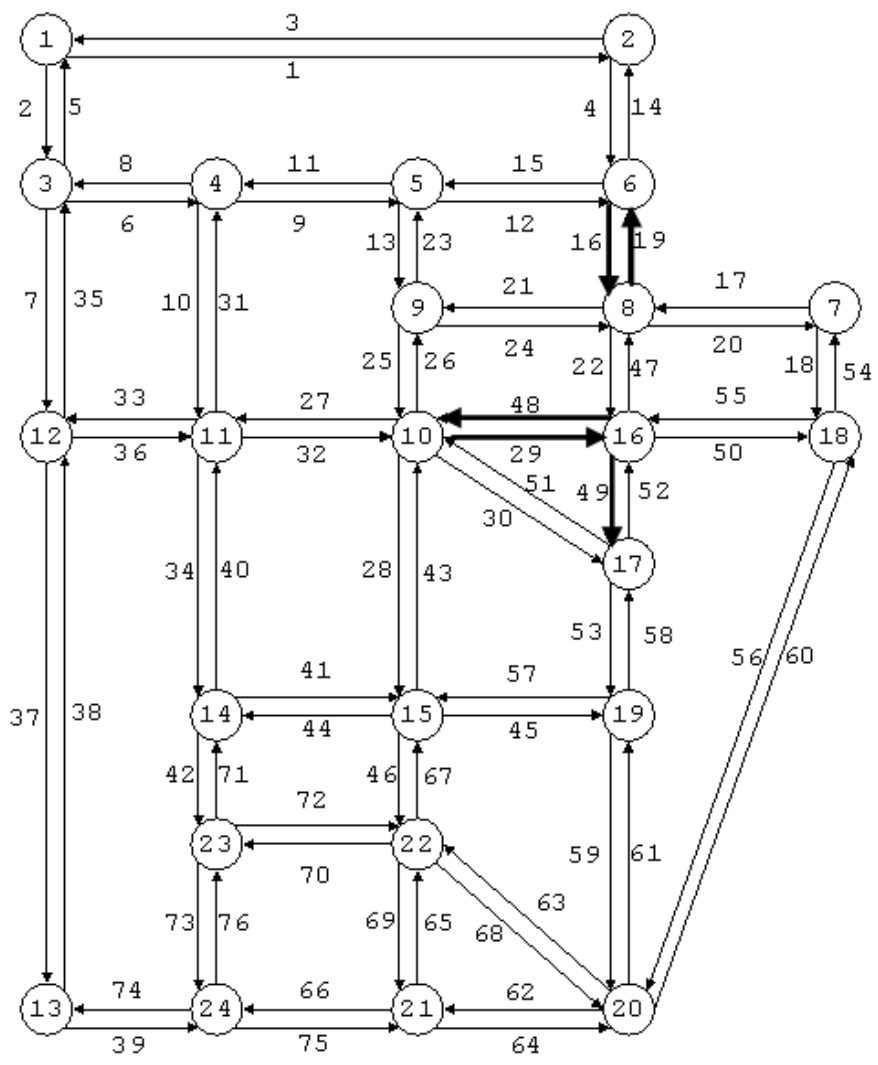

\section{Figure 4. Sioux-Falls Network with the top 5 Congested Links}

\subsection{The Sioux-Falls Network}

The Sioux-Falls network, as shown in Figure 4, is widely used in transportation studies. It has 24 nodes and 76 links, and other parameters can be obtained from http://www.bgu.ac.il/ bargera/tntp/.

We first assign the traffic in the network following UE principle. Links 19, 16, 48, 29, 49 are the most congested links in terms of the ratio of flow and capacity $(2.557,2.550,2.280$, 2.276, 2.237, respectively). These five links are indicated by thick lines in Figure 4. Hence, we set $\bar{A}=\{16,19,29,48,49\}$, and possible toll levels $I_{a}=\{0,1\}$, and $\tau_{a}^{0}=0, \tau_{a}^{1}=0.8$ for all $a \in \bar{A}$.

The results are shown in Table 5. The solutions with and without the consideration of demand uncertainty are different. Considering demand uncertainty, the relative efficiency is improved by $(2.10 \%-1.88 \%) / 1.88 \%=12 \%$. It should be noted that since the Sioux-Falls network has 76 links, and only 5 links are tolled (second-best pricing), we cannot expect the relative efficiency to be near 1 .

Table 5. Importance of Considering Demand Uncertainty in the Sioux-Falls Network

\begin{tabular}{|c|c|c|}
\hline Strategy & Use the mean demand & Consider demand uncertainty \\
\hline Optimal toll & $\begin{array}{c}\text { No toll on link } 48 \text { and a toll of } 0.8 \\
\text { on links } 16,19,29,49\end{array}$ & $\begin{array}{c}\text { No toll on link } 16 \text { and a toll of } 0.8 \\
\text { on links } 19,29,48,49\end{array}$ \\
\hline Relative efficiency & $1.88 \%$ & $2.10 \%$ \\
\hline
\end{tabular}




\section{Conclusions}

We have examined a new and practical second-best pricing problem with uncertain demand. This problem can be formulated as a stochastic mathematical program with equilibrium constraints. In view of the problem structure, we develop a tailored global optimization algorithm. This algorithm incorporates a sample average approximation scheme, a relaxation-strengthening method, and a linearization approach. The proposed global optimization algorithm is applied to three networks: a two-link network, a seven-eleven network and the Sioux-Falls network. The results demonstrate that using a single fixed estimation of future demand may overestimate the future system performance. Moreover, the optimal toll obtained by using the mean demand value may not be optimal considering demand uncertainty. The proposed global optimization algorithm explicitly captures demand uncertainty and yields solutions that outperform those without considering demand uncertainty.

In this study the toll information in constant, and hence can easily be known by users. The demand information may change from day to day: for instance, one pattern on Monday, one pattern on Tuesday, etc. Since the purpose of congestion pricing is to alleviate congestion during peak hours, which are the time for commuting to work and back home, it is reasonable to assume that users have enough experience about the traffic conditions (they travel to and from work every day). That is the rationale behind assuming that users have full information.

Although the core elements of the paper require a high level of mathematical expertise, the fundamental idea of our model is simple: Since there are many demand scenarios, we try to find a toll that is the best for the average outcome of all these demand scenarios. However, it may not be easy to understand this idea correctly. In practice, transport authorities collect OD travel data for many days. Evidently, the collected data on different days would be different, and a natural (whereas wrong) approach is to use the average travel demand to replace the underlying stochastic demand. As demonstrated by our paper, the optimal toll obtained by using the mean demand may not be optimal considering demand uncertainty. In other words, using the mean demand may lead to suboptimal solutions. That is a rule on which special attention should be paid by practitioners when setting tolls.

To implement the model in reality, the transport authority needs to do the following: (i) Determine a set of candidate roads for toll pricing; (ii) Collect the origin-destination travel information on different days; (iii) Apply the proposed model and algorithm to calculate the optimal toll charge on each candidate road; (iv) Publish the toll information in advance and set up toll gantries to collect tolls.

There are a few research directions that we will explore in future. First, in this study we assume homogeneous travelers in the network with the same value of time (VOT). In reality travelers with higher income generally have a higher VOT. Moreover, different OD pairs may have different compositions of VOTs (for example, if the destination is a central business district with many banks, then the most traveler have a very high VOT). Second, besides efficiency considerations, incorporating travelers of different VOTs would lead to another important issue: the equity between different traveler groups. Equity plays a central role in effectiveness, acceptability and ease of implementation of toll pricing. Therefore, how to incorporate equity in pricing is an interesting research topic. 


\section{References}

[1] C. and K. Subprasom, "Analysis of regulation and policy of private toll roads in a build-operatetransfer scheme under demand uncertainty", Transportation Research Part A, vol. 41, no. 6, (2007), pp. 537-558.

[2] L. M. Gardner, A. Unnikrishnan and S. T. Waller, "Solution methods for robust pricing of transportation networks under uncertain demand", Transportation Research Part C, vol. 18, no. 5, (2010), pp. 656-667.

[3] S. Lawphongpanich and D. W. Hearn, "An MPEC approach to second-best toll pricing", Mathematical Programming, vol. 101, no. 1, (2004), pp. 33-55.

[4] H. Li, M. C. J. Bliemer and P. H. L. Bovy, "Network reliability-based optimal toll design", Journal of Advanced Transportation, vol. 42, no. 3, (2008), pp. 311-332.

[5] L. N. Liu and J. F. McDonald, "Economic efficiency of second-best congestion pricing schemes in urban highway systems", Transportation Research Part B, vol. 33, no. 3, (1999), pp. 157-188.

[6] W. Liu, H. Yang and Y. Yin, "Traffic rationing and pricing in a linear monocentric city", Journal of Advanced Transportation, doi: 10.1002/atr.1219, (2013).

[7] Z. Liu, "Probit-based Stochastic User Equilibrium and Their Applications in Congestion Pricing", $\mathrm{PhD}$ Thesis, National University of Singapore, (2011).

[8] Z. Liu, Q. Meng and S. Wang, "Speed-based toll design for cordon-based congestion pricing scheme", Transportation Research Part C, vol. 31, (2013), pp. 83-98.

[9] Z. Liu, Y. Yan, X. Qu and Y. Zhang, "Bus stop-skipping scheme with random travel time", Transportation Research Part C, vol. 35, (2013), pp. 46-56.

[10] P. Luathep, A. Sumalee, W. H. K. Lam, Z. C. Li and H. K. Lo, "Global optimization method for mixed transportation network design problem: A mixed-integer linear programming approach", Transportation Research Part B, vol. 45, no. 5, (2011), pp. 808-827.

[11] W. K. Mak, D. P. Morton and R. K. Wood, "Monte Carlo bounding techniques for determining solution quality in stochastic programs", Operations Research Letters, vol. 24, (1999), pp. 47-56.

[12] Q. Meng and Z. Liu, "Trial-and-error method for congestion pricing scheme under side-constrained probit-based stochastic user equilibrium conditions", Transportation, vol. 38, no. 5, (2011), pp. 819843.

[13] Q. Meng and Z. Liu, "Impact analysis of cordon-based congestion pricing scheme on mode-split of bimodal transportation network", Transportation Research Part C, vol. 21, no. 1, (2012), pp. 134147.

[14] Q. Meng, Z. Liu and S. Wang, "Optimal distance tolls under congestion pricing and continuously distributed value of time", Transportation Research Part E, vol. 48, no. 5, (2012), pp. 937-957.

[15] Q. Meng and X. Qu, "Bus dwell time estimation at bus bays: A probabilistic approach", Transportation Research Part C, vol. 36, (2013), pp. 61-71.

[16] T. Nagae and T. Akamatsu, "Dynamic revenue management of a toll road project under transportation demand uncertainty", Network Spatial Economics,vol. 6, no. 3-4, (2006), pp. 345-357.

[17] A.C. Pigou, "The Economics of Welfare", Macmillan and Co., London, (1920).

[18] E.T. Verhoef, "Second-best congestion pricing in general networks. Heuristic algorithms for finding second-best optimal toll levels and toll points", Transportation Research Part B, vol. 36, no. 8, (2002), pp. 707-729.

[19] B. Verweij, S. Ahmed, A. J. Kleywegt, G. Nemhauser and A. Shapiro, "The sample average approximation method applied to stochastic routing problems: a computational study", Computational Optimization and Applications, vol. 24, (2003), pp. 289-333.

[20] S. T. Waller, J. L. Schofer and A. K. Ziliaskopoulos, "Evaluation with traffic assignment under demand uncertainty", Transportation Research Record, vol. 1771, (2001), pp. 69-74.

[21] S. Wang, Q. Meng and Z. Liu, "Fundamental properties of volume-capacity ratio of a private toll road in general networks", Transportation Research Part B, vol. 47, (2013), pp. 77-86.

[22] H. Yang, W. Xu and B. Heydecker, "Bounding the efficiency of road pricing", Transportation Research Part E, vol. 46, no. 1, (2010), pp. 90-108.

[23] H. Yang and X. Zhang, "Optimal toll design in second-best link-based congestion pricing", Transportation Research Record, vol. 1857, (2003), pp. 85-92.

[24] H. Yang, X. Zhang and Q. Meng, "Modeling private highways in networks with entry-exit based toll charges", Transportation Research Part B, vol. 38, no. 3, (2004), pp. 191-213.

[25] X. Zhang, and B. van Wee, "Enhancing transportation network capacity by congestion pricing with simultaneous toll location and toll level optimization", Engineering Optimization, vol. 44, no. 4, (2012), pp. 477-488. 
[26] X. Zhang and H. Yang, "The optimal cordon-based network congestion pricing problem", Transportation Research Part B, vol. 38, no. 6, (2004), pp. 517-537.

\section{Authors}

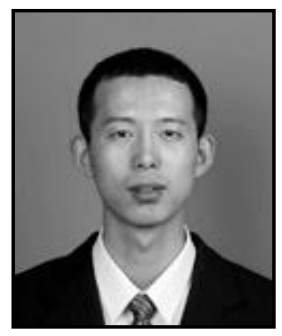

Shuaian Wang, obtained his $\mathrm{PhD}$ in Transportation Engineering at the National University of Singapore in 2012. His research interest is focused on maritime container transportation, intermodal freight systems, and transportation and traffic engineering.

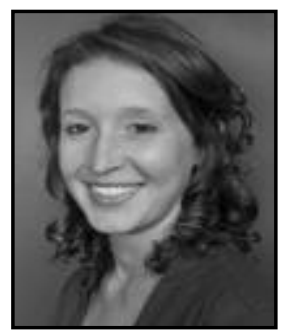

Lauren M. Gardner, is a lecturer of Transport Engineering at the University of New South Wales.

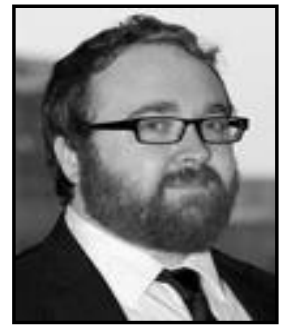

S. Travis Waller, is the Evans \& Peck Professor of Transport Innovation at the University of New South Wales. 